\section{Is Natural Selection all Metaphor?}

The Duke of Argyll, in his reply to Mr. Herbert Spencer, says " in the Darwinian theory there is no selector" (NATURE, February 2, p. 317). Though we have not yet discovered a principle or factor which plays the part of the breeder in nature, it by no means follows that " natural selection" is "all metaphor," nor yet, as has been often stated, an altogether misleading phrase. The rôle of the breeder or artificial selector is, I believe, often misunderstood. If we consider what the art of breeding mainly consists in, we may come to the conclusion that even the phrase " artificial selection" is, to a considerable extent, misleading and metaphorical. It seems to me the art of breeding consists mainly in two things, viz. (I) producing prepotency, and (2) preventing intercrossing. Prepotency is produced and maintained by inbreeding. The object of preventing intercrossing is to arrest, as far as possible, variation and reversion. If it can be shown that in nature prepotency often arises either as a sport or through inbreeding, and that prepotency by arresting the "swamping effects of intercrossing" plays the part of the fences of the breeder and the cages of the fancier, we shall be justified in looking upon prepotency as a " selector," and in finding more than metaphor in the phrase "natural selection." We already know that amongst insects a sport may displace the parent form; and if, instead of searching for evidence of inter sterility as suggested by Romanes, we search diligently for evidence of prepctency, we may ere long discover the " selector" - the factor that in nature, under the control of utility, plays the part of the breeder.

J. C. EWART.

\section{Geometry versus Euclid.}

To a great many people the assertion that the teaching of geometry from Euclid's book in the schools-and especially in the preparatory schools-is a positive hindrance to the teaching of science will be regarded as paradoxical, if not, indeed, erroneous. Yet I do make the assertion; and I base my confidence in its truth mainly on the experience which I have gained as an examiner of boys who have finished their school education.

Geometry is about the oldest of the sciences, and Euclid's venerable work bears all the characteristics of a book compiled at a remote time when such science as existed was a kind of mysterious possession in the hands of a few experts to whom intricate technicality of language was (as Swift would say) a principle of great emolument. The inventor of a new science is only too prone to build it up with an elaborate and technical system of definition and nomenclature, hoping thereby to emphasise its importance and to cultivate a wholesome awe in the uninitiated. In this way is established a particular kind of jargon which becomes distinctive of the science, and of its professional exponents.

The growth of such a system is well exemplified in other domains than that of science. For example, there is not, I think, any game in vogue in England which possesses such an elaborate technical jargon as that of golf, and the rule which is always observed in such matters is here strictly recognisedviz. the less the intrinsic merit of the subject, the more elaborate the accompanying jargon.

We are all very familiar with the Euclid jargon. Some of us, indeed, have somehow come to believe that no proof of a proposition can possibly be valid unless it is presented in this orthodox form.

A modern Euclid for the use of schools is sometimes a model of soul-destroying systematisation. I have before me such a work in which the process of arriving at the conclusion that two angles of a triangle are equal if the sides opposite to them are equal, reminds me of the process of walking across a lawn over the surface of which have been stretched innumerable threads in various directions for the purpose of tripping up the unwary.

The number of heads under which a well-taught modern boy will arrange the most simple proposition is wonderful : "general enunciation," " particular enunciation," " hypothesis," "construction," "demonstration," "conclusion " must all figure, or else the proof is " no good." Only a boy who has been careless says, "if two triangles have three sides of the one equal to three sides of the other, the triangles are equal in all respects" - a very simple truth which I received once in the following form from a boy who was much more careful of the orthodox jargon: "if two triangles have two sides of the one respectively equal to two sides of the other, each to each, and likewise also their bases, or third sides, equal, then shall the three angles of the one triangle be equal to the three angles of the other triangle, and the triangles shall be equal in every respect."

Observe that in the Euclid jargon nothing ever simply "is" -it always " shall be."

In finding fault with Euclid as a book for beginners I have, of course, no right to charge it with the enormous number of definitions, and the dissertations on the various kinds of propositions ("positive," "contra-positive," \&c.) which some of the school-books set right in front of the beginner before the first proposition of the first Book is reached.

Still, it is by no means the paragon of logical clearness that it is commonly alleged to be. Take, for instance, its very first definition: "a point is that which has no parts." This is an excellent definition of absolute nonentity, but not of anything that can be pictured in the mind. Some editors of Euclid, feeling that there is something wanting in this definition, have (they think) vastly improved it by saying that "a point is that which has position but no magnitude"-as if position is more easily grasped than point. Then again (still at the threshold of the subject) the beginner is taught to believe that he is getting a very definite conception of a right line in the definition, "a right line is that which lies evenly between its extreme points" -as if the meaning of "evenly" is at once beyond question.

But of all the elementary conceptions in Euclid that of an angle is the one which most puzzles a beginner, and remains unrealised for the longest time. "An angle is the inclination of two straight lines to one another." Here again we have one obscure term defined by another equally obscure ; and we know by experience that, unless the conception is presented in a very different way, the obscurity will be permanent.

Moreover, it is possible to point out a self-contradiction in Euclid. Thus his definition of a circle makes it to be a disc"a circle is a plain figure bounded by one line called the circumference"-so that, clearly, the whole of the space inside (or, possibly, outside) the circumference is the circle, whose mere boundary is the circumference; and, if so, two circles can, of course, intersect in an infinite number of points-over an extensive area, in fact; but this is contradicted by Euclid in the tenth proposition of Book III., according to which one circle cannot intersect another in more than two points.

These, it may be admitted, are comparatively minor considerations, and the defects might be corrected by judicious teaching.

It is chiefly in the way in which the fifth and sixth Books of Euclid are apprehended by boys that the necessity for a change in the system of teaching is to be seen.

Those mediæval technicalities "duplicate ratio," "subduplicate ratio,". "sesquiplicate ratio," and some others are drummed into the heads of boys as if they were terms of the utmost scientific importance. What mathematician ever uses such terms, or even thinks of them in his investigations?

The simple and extremely important fact that the areas of two similar figures are to each other as the squares of corresponding linear dimensions is presented to the beginner in the nineteenth proposition of the sixth Book in the words "similar triangles are to one another in the duplicate ratio of their homologous sides"-a statement which is singularly deficient in accuracy inasmuch as it omits to say precisely what two qualities or quantities connected with the triangles are thus related (colours, shapes, sizes, or what?); and the result is absolute confusion in the minds of a very large number of boys

Let me illustrate this by a few bona fide examples. In reply to the question, "What are similar triangles, and what is the relation between their areas?" the following answers were received :-

(I) A triangle is similar to another triangle when their sides are proportional, and when the homologous sides of one are in duplicate ratio to the homologous sides of the other.

(2) If two triangles have the sides about an angle in each proportional and the other angles of the same affection, the triangles are similar. Similar triangles are proportional to the bases on which they stand, and are to one another in the duplicate ratio of their homologous sides.

(3) Similar triangles are those which are equal in area to each other and are in the same proportion to each other as the duplicate ratio of their homologous sides.

(4) When the angles are similar the areas are similar, when the areas are similar the angles are similar, when the sides are similar the areas are similar. 